\title{
Studies of the Centaurus cluster
}

\section{A catalogue of galaxies in the central region of the Centaurus cluster $^{\star}$}

\author{
H. Jerjen ${ }^{1, \star \star}$ and A. Dressler ${ }^{2}$ \\ 1 Astronomisches Institut der Universität Basel, Venusstrasse 7, CH-4102 Binningen, Switzerland \\ 2 Carnegie Observatories, 813 Santa Barbara St., Pasadena, CA 91101, U.S.A.
}

Received April 3; accepted October 24, 1996

\begin{abstract}
The central region of the Centaurus cluster was surveyed on a film copy of a high-resolution photographic plate taken with the 100-inch du Pont Las Campanas telescope. From visual inspection of the film, a large number of cluster galaxy candidates were identified down to a limiting magnitude of 21.5 in $B$. Between cluster members and background objects were distinguished by applying morphological criteria established in previous studies of the Virgo and Fornax clusters. Following the morphological classification of each member, the images of all 296 cluster galaxies on a SRC deep-blue sky survey plate were digitized, processed and analysed with programs written in the image processing package MIDAS. Aperture photometry yielded growth curves and total instrumental magnitudes of the galaxies. The magnitudes were calibrated with independent photometric data and subsequently transformed into standard $B$-magnitudes. We further established a set of structure parameters for each cluster galaxy: effective surface brightness, effective radius, central exponential surface brightness, and scale length. For the ellipticals we additionally determined the shape parameters of the light profiles.
\end{abstract}

Key words: galaxies: clusters: Centaurus cluster - galaxies: fundamental parameters — galaxies: photometry - catalogs

Send offprint requests to: H. Jerjen

* The catalogue (CCC) is only available in electronic form at the CDS via anonymous ftp to cdsarc.u-strasbg.fr (130.79.128.5) or via http://cdsweb.u-strasbg.fr/ Abstract.html

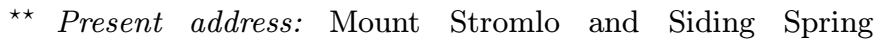
Observatories, Private Bag, Weston Creek PO, ACT 2611, Canberra, Australia, e-mail: jerjen@mso.anu.edu.au

\section{Introduction}

In the last ten years, two comprehensive studies of dwarf galaxy populations in galaxy clusters have been carried out based on photographic plate material taken at the Las Campanas du Pont 100-inch telescope. This telescope had a unique imaging capability, being equipped with a wide field $\left(1.5^{\circ} \times 1.5^{\circ}\right)$ camera for $50 \mathrm{~cm} \times 50 \mathrm{~cm}$ high resolution (10.9 arcsecs $\mathrm{mm}^{-1}$ ) photographic glass plates (Bowen \& Vaughan Jr. 1973). Because it was the only telescope worldwide offering such a facility, it became of great importance for the early exploration of dwarf populations in galaxy aggregations like clusters and groups.

The first surveyed cluster was the nearest cluster in Virgo, which also is the dominating structure of the Local Supercluster. Based on the Virgo Cluster Catalogue (VCC, Binggeli et al. 1985), Binggeli, Sandage and collaborators presented a series of papers on different aspects of this cluster and its member galaxies (Binggeli et al. 1984, 1987; Sandage \& Binggeli 1984; Sandage et al. 1985a,b) which significantly improved the knowledge in several fields of research, e.g. morphological classification of dwarf galaxies, their photometric and physical properties, type-specific luminosity functions of galaxies, variation of galaxy type mixture in dependence of the environmental density, substructure in clusters of galaxies.

Ferguson (1989), in collaboration with Sandage, carried out a dwarf galaxy study in the Fornax cluster a few years later. This less rich cluster lies roughly at the same distance to the Local Group as the Virgo cluster. Ferguson \& Sandage (1988) investigated the cluster luminosity function (LF) and compared it with the LFs of Virgo and five nearby groups of galaxies (Ferguson \& Sandage 1991). The analysis of the morphological type distributions revealed the extension of the morphology-density relation for cluster galaxies (Dressler 1980a; Binggeli et al. 1987) to group environments. 
Many interesting results have been found for the different galaxy types, in particular for the dwarf galaxies, based on the data of the two cluster populations in Virgo and Fornax. However due to the small number of only two cluster studies, it is still debated whether the observations reflect "cosmic" properties or they are characteristic for only a specific cluster population. To follow up this question, it was planned to carry out a further dwarf population study in a more distant cluster which would be as extensive and detailed as the previous two cluster studies. Our intention was to take photographic plates with the du Pont telescope but the wide-field camera was no longer available. We therefore worked with existing du Pont plates which were part of the material used to establish the morphology-density relation for classical Hubble types (Dressler 1980b).

Upon a visual examination of high quality film copies of ten du Pont plates each covering one galaxy cluster with a redshift between $0.01<z<0.03$, the Centaurus cluster at $c z \sim 3300 \mathrm{~km} \mathrm{~s}^{-1}$ appeared as the most promising cluster for our project. An excellent photographic exposure, taken under good seeing conditions, was available. Additionally, there are several scientific reasons in favour of this cluster. The giant galaxies in Centaurus show a pronounced bimodal distribution in the sky, pointing to a prominent substructure feature. This brings up the question about the distribution of the dwarf galaxies. Moreover, a remarkable bimodal velocity distribution, known as Cen30 and Cen45, has been reported by Lucey et al. (1980) and Lucey et al. (1986). Is it caused by the dynamics of an infalling group of galaxies (Cen45) into the main cluster component (Cen30) both belonging to the same young cluster which is still in the stage of formation, or is the gravitational force of a nearby "Great Attractor" (Lynden-Bell et al. 1988) responsible for the unusual velocities? Amongst others, these questions will be of special interest within our study.

All results of our Centaurus cluster survey are collected in a series of five papers. The present Paper I describes the technical aspects we used to establish the sample galaxies of the Centaurus Cluster Catalogue and to acquire structure parameters for each galaxy. The subsequent papers of the series will appear in the main journal of A\&A:

In Paper II we discuss the distributions of the individual Hubble types within the surveyed cluster region. We report the detection of a prominent zone of very low galaxy density and explore the galaxy type mixture. After deriving the clusterspecific parameters, the Centaurus galaxy population is compared to those of the Virgo and the Fornax cluster. Recent results of the Cen $30 / 45$ puzzle have been taken into account to infer consequences on the real spatial distribution of the Centaurus cluster galaxies. This leads to a new distance of Centaurus relative to Virgo which is discuss within a cosmological context.

In Paper III the luminosity functions are presented for the complete cluster population as well as for many individual Hubble types. Their properties are discussed and compared to the corresponding LFs in Virgo. It is suggested that the individual LFs of S0's, spirals, and dE\&dS0's are good distance indicators. Furthermore, we give new reasons which definitively call into question the reliability of the Schechter parameter $M^{*}$ to measure cluster distances.

Paper IV is dedicated to the systematic photometric properties of the early-type dwarf galaxies. Moreover, we use two relations to establish a new distance for Centaurus and Fornax relative to Virgo, respectively.

Finally, in Paper V a 2D-substructure analysis is carried out on the three cluster populations of Centaurus, Virgo, and Fornax. For the first time dwarf galaxies are taken into account for this purpose.

\section{Identification and classification of galaxies}

Our field of survey corresponds to the field of the 20-inch du Pont plate ID: CD14, taken on Eastman blue emulsion 103a-O, baked in forming gas and exposed for one hour on the 100-inch du Pont reflector at Las Campanas. The plate includes the central part of the Centaurus cluster covering an area of $2.2 \square^{\circ}$ bound by $12^{\mathrm{h}} 44^{\mathrm{m}} 40^{\mathrm{s}}<\mathrm{RA}(1950)$ $<12^{\mathrm{h}} 52^{\mathrm{m}} 40^{\mathrm{s}}$ and $-41^{\circ} 40^{\prime}<\operatorname{Dec}(1950)<-40^{\circ} 12^{\prime}$.

We carried out a visual search for all non-stellar objects with apparent diameters larger than $10^{\prime \prime}=0.9 \mathrm{~mm}$ on the high-quality film copy of the Centaurus du Pont plate ( $\sim 0.15 \mathrm{~mm}$ on a Schmidt plate). Using a magnifier more than 1100 galaxies have been detected down to this diameter limit. In order to find very low-surfacebrightness galaxies and to rule out plate flaws from our sample, we additionally employed film copies of the deeper, but low-resolution Schmidt plates SRC 322J and 323J (GG395-IIIa-J, 70 minutes exposure time) from the European Southern Observatory/Science Research Council (ESO/SRC) Southern Sky Survey.

The criteria we applied to subdivide the sample galaxies into Centaurus cluster members and other galaxies (background) are those worked out by, and described in, Binggeli et al. (1985). This means we had to take into account the surface brightness, the resolution of the galaxy in stellar content, and - if available - the radial velocity. Ferguson (1989) already applied successfully these criteria in his survey of the Fornax cluster. Meanwhile it was shown for the Virgo cluster (Binggeli et al. 1993; Drinkwater et al. 1996) that such morphological guesses are in excellent agreement with redshift data.

We estimate the likelihood of each "cluster member" to be in the cluster by assigning a probability of membership in three steps of $50 \%, 75 \%$ or $100 \%$ depending on how strongly the galaxy fulfils the above mentioned criteria. Thus the initial survey sample ended in 4 different classes whose numbers are listed in Table 1.

The process of cluster/background subdivision and cluster membership assignment was additionally 
Table 1. Cluster membership code with numbers

\begin{tabular}{cccr}
\hline Class & Meaning & Membership & \multicolumn{1}{c}{$n$} \\
\hline 1 & definite member & $100 \%$ & 198 \\
2 & likely member & $75 \%$ & 32 \\
3 & possible member & $50 \%$ & 66 \\
4 & background & $0 \%$ & $>800$ \\
\hline
\end{tabular}

facilitated by the fact that the surveyed region of the Centaurus cluster is nearly free of field or nearby background cluster galaxies which could strongly contaminate the Centaurus cluster sample (or even completely impede the survey). Besides rather few foreground objects, a first galaxy concentration is located behind Centaurus at a redshift of $c z \sim 9000 \mathrm{~km} \mathrm{~s}^{-1}$ (e.g. Dickens et al. 1986; Stein 1994) or roughly 3.5 times the distance to the surveyed cluster. This fact makes us quite confident that our selection of the 296 cluster members is stable against background contamination. All uncertainties are expected to be covered by the given membership probabilities.

Galaxies of the final cluster sample were morphologically classified by the visual comparison of their images on the du Pont and Schmidt films with the reference galaxies of the extended Hubble classification system. These reference galaxies are illustrated in the Hubble Atlas (Sandage 1961), the Revised Shapley-Ames Catalogue (Sandage \& Tammann 1981), the Atlas of Virgo Cluster Spiral Galaxies (Sandage et al. 1985a), and in the Atlas of Virgo Cluster Dwarf Galaxies (Sandage \& Binggeli 1984). For the particular distinction between dwarf ellipticals ( $\mathrm{dE}$ ) and dwarf S0 (dS0) galaxies the explanation in Binggeli \& Cameron (1991) was very helpful. Table 2 lists the frequency of the main Hubble types in our cluster catalogue as non-weighted (Cols. 2 and 3) and membership-weighted numbers (Cols. 4 and 5).

In general, the detection of a galaxy as well as the accuracy of a morphological classification strongly depend on the Hubble type, the inclination and the angular size. The most crucial points are summarized here:

Low luminosity elliptical galaxies (exemplified by M32) are very compact and in appearance comparable to the seeing disk of a star. Only the missing diffraction cross typical for point sources gives evidence for a nonstellar object. The Centaurus du Pont plate has a magnitude limit for crossed stars of about $B_{\mathrm{T}}=15.0$ or $\sim-19.0$ in absolute $B$-magnitude. On the other hand, a M32-type galaxy typically has $M_{B_{\mathrm{T}}} \sim-16$ which explains why the detection of such objects in our survey is a matter of chance. Nevertheless, our galaxy catalogue contains one strong candidate: object CCC70 is a low luminosity elliptical with $M_{B_{\mathrm{T}}}=-18.12$ and a characteristic high mean surface brightness. We will discuss its properties in Paper IV in more details (Jerjen \& Binggeli 1997).
Early-type dwarfs (dE\&dS0) exhibit no active star formation and thus are detected primarily based on their star density (= surface brightness). For this galaxy type the surface brightness at a specific radius is correlated with the total magnitude of the galaxy (Binggeli \& Cameron 1991; Paper IV). As we demonstrate in Paper IV the survey limit is $9^{\prime \prime}$ in diameter at the isophote of $26.5 \mathrm{~B} \mathrm{arcsec}^{-2}$, which corresponds to a completeness limit of about $B_{\mathrm{T}}=18.5$ for the dE\&dS0 galaxies.

Table 2. Hubble type frequencies as non-weighted and membership-weighted numbers

\begin{tabular}{c|rrcr}
\hline Type & $n$ & $\%$ & $n$ & $\%$ \\
$(1)$ & $(2)$ & $(3)$ & $(4)$ & $(5)$ \\
\hline All & 296 & 100 & 258 & 100 \\
E & 17 & 6 & 16.25 & 6 \\
S0 & 34 & 11 & 32 & 12 \\
Sp & 30 & 10 & 27 & 11 \\
Im\&BCD & 55 & 19 & 40.75 & 16 \\
dE\&dS0 & 160 & 54 & 142 & 55 \\
\hline Im & 49.5 & 17 & 37.75 & 15 \\
BCD & 5.5 & 2 & 3 & 1 \\
dE & 142 & 48 & 128.25 & 50 \\
dS0 & 18 & 6 & 13.75 & 5 \\
\hline
\end{tabular}

In contrast to the early-type dwarfs, Im's exhibit a wide range in surface brightness at a given absolute magnitude due to the sensitivity to star-formation activity. This naturally biases detection against inactive Im's which should be taken into account if one works with Im samples. On the other hand, BCD's (Blue Compact Dwarfs) are compact extragalactic HII regions. Their bright irregular shape gives a high detection probability. But the appearance of a cluster BCD can be very similar to a star formation region of a more distant Im galaxy or a field BCD which makes the definite assignment of a BCD difficult in most cases. Nevertheless, only recently Drinkwater et al. (1996) confirmed with new redshift data that $97 \%$ of a morphological ground based sample of "background" BCD's from the Virgo Cluster Catalogue (Binggeli et al. 1985) are indeed not members of the Virgo cluster.

Figure 1 gives an overview of the surveyed cluster region with the cluster members subdivided into the five main Hubble types E, S0, Sp, Im\&BCD, and dE\&dS0. All cluster members, with their membership probability and detailed Hubble type, are listed in the Centaurus Cluster Catalogue (hereafter CCC).

\section{Photometry}

After the acquisition of the cluster sample our goal was to determine the apparent $B_{\mathrm{T}}$-magnitude and other fundamental parameters for each of the program galaxies. 


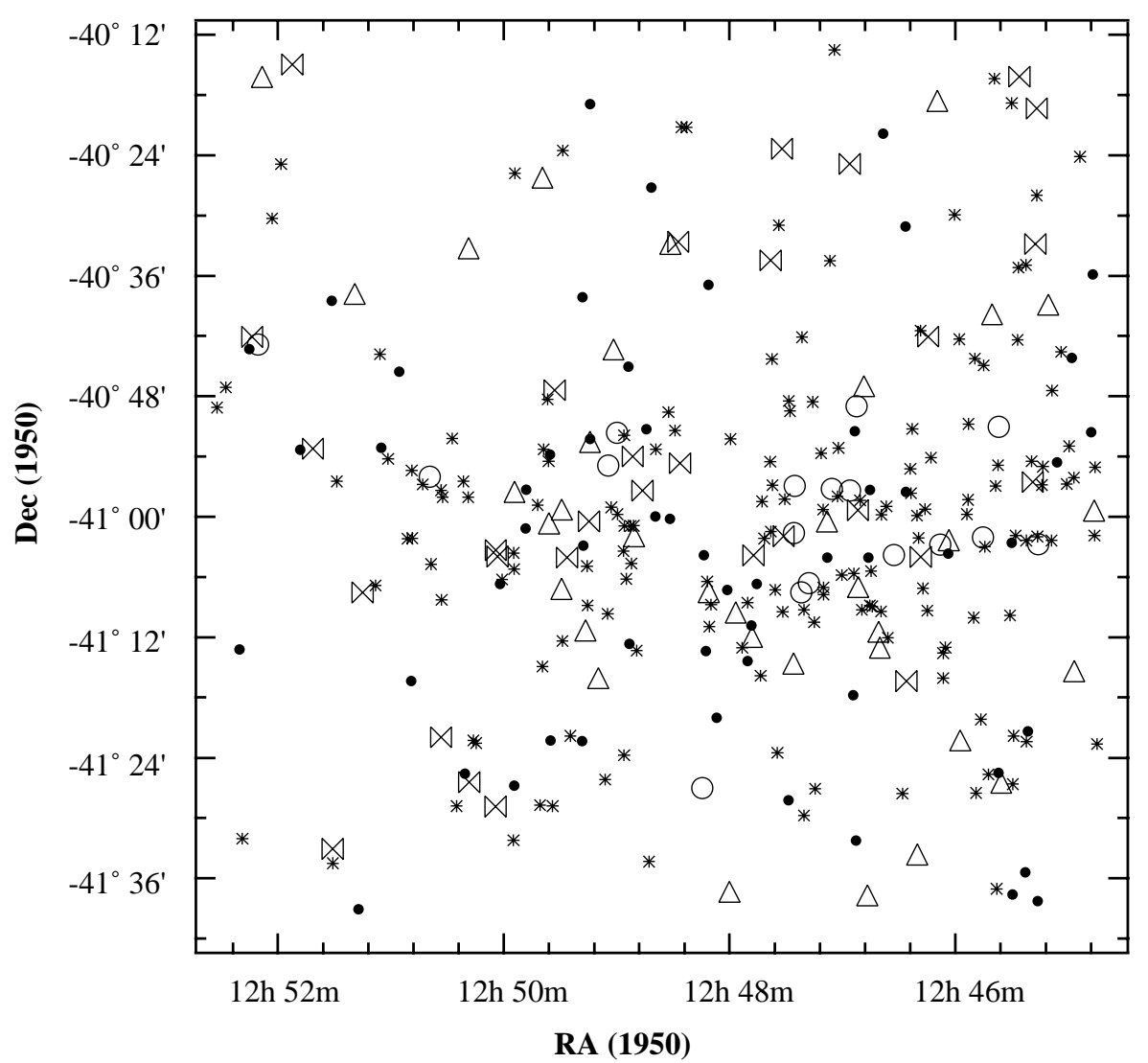

Fig. 1. The survey area covered by the du Pont plate is shown. The cluster members have been divided into five different symbols $(\circ, \triangle, \bowtie, \bullet, *)$ according to the five main Hubble types E, S0, Sp, Im\&BCD, and dE\&dS0

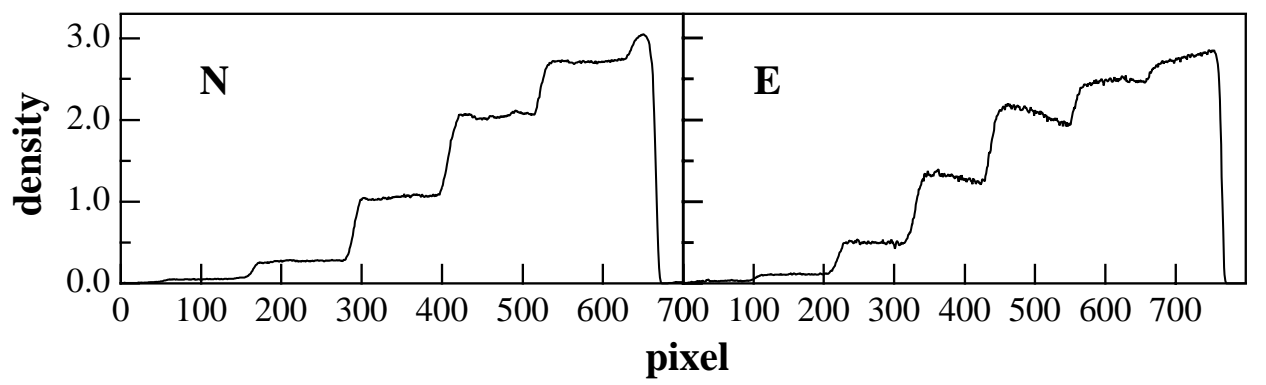

Fig. 2. Averaged density profiles of the northern and eastern sensitometer marks of the SRC 323J Schmidt plate

Unfortunately, the du Pont film (and plate) was not suitable for this purpose due to the lack of sensitometer marks. Thus, we decided to do photometry with a first generation glass copy of the Schmidt plate SRC 323J from the archive of the ESO headquarters in Garching. The plate is equipped with 7 step sensitometer marks on its northern and eastern margin allowing to establish the density-tointensity transformation.

\subsection{Tracings}

The part of the Schmidt plate 323J which coincides with the surveyed field was digitized with the Perkin Elmer PDS 1010A 2-D micro-densitometer system at the ESO headquarters. After the calibration of the instrument with an unexposed area (plate fog) at the edge of the plate, 16 subfields covering the survey region were scanned in the density mode with a 12 bit $\mathrm{A} / \mathrm{D}$ converter. $25 \mu \mathrm{m}$ were chosen for the size of the squared beam aperture as well 
as for the step size of the scan. With a plate scale of 67.5 arcsecs $/ \mathrm{mm}$ and a seeing of 2.3 arcsecs for the particular ESO Sky Survey plate, this led to a slight oversampling. The seeing value was derived from the faintest stars on the plate. The scanning velocity is inversely correlated to the maximum reliable density that can be registered by the system. We chose $v_{\text {scan }}=20 \mathrm{~mm} \mathrm{~s}^{-1}$ enabling us to measure densities up to $D=3.1$. This density limit is somewhat higher than the expected saturation level due to photographic processes at $D \sim 3$ or $\mu_{B} \sim 21.5 \mathrm{~B} \operatorname{arcsecs}^{-2}$. In this way, the dynamical range of the plate was covered best.

\subsection{Characteristic curve}

Additionally to the science field the two strips of sensitometer marks were fully traced. In Fig. 2 we show plots of their smoothed profiles averaged over 50 scanning rows. While the northern strip yields very well defined density steps, with the exception of the highest, the average profile of the eastern strip is of only poor quality. Several density steps show gradients superposed by strong defects. No accurate measurements of these density values were possible. This circumstance together with the fact that no $\log (I)$ values were available for the eastern projector, neither in the UKSTU-Handbook (Tritton 1983) nor from the Royal Observatory in Edinburgh, constrained us to work with the northern marks only.

To establish the characteristic curve of the plate we used the bi-logarithmic formula proposed by Llebaria \& Figon (1981):

$\log (I)=A * \log \left(D_{\text {sat }}-D\right)+B * \log \left(D-D_{\text {fog }}\right)+C$,

where $E=I \cdot t$ of the original formula is replaced by the more convenient quantity $I=E / t$. The different parameters have the following meaning: $I$ and $D$ are the relative intensity and the density, respectively. $D_{\text {sat }}$ is the density at which the photographic plate becomes saturated, $D_{\text {fog }}$ is the zero-point density of the photographic emulsion, and $C$ is the zero point. The analytic curve was $\chi^{2}$-fitted at the points defined by the density values of the northern step marks and their corresponding $\log (I)$-values (Tritton 1983). As already mentioned above, the highest step of the northern sensitometer mark was not very well defined. Consequently, a first fit with this original density value was unsatisfactory, i.e. the derived value of the fit parameter $D_{\text {sat }}$ was lower than the highest scanned density found on the plate. To overcome this problem empirically we applied a $5 \%$ correction to the highest step value from 3.039 to 3.179 in order to reach the highest plate density. Figure 3 shows the curve which fits best the finally used data points and in Table 3 we list the bestfitting parameters.

For an external quality check of the density-tointensity transformation Bender (from the CCD work of Bender 1994) kindly provided us with the major
Table 3. Parameters of the characteristic curve

\begin{tabular}{lr}
\hline Parameter & Value \\
\hline$A$ & -0.555 \\
$D_{\text {sat }}$ & 3.689 \\
$B$ & 0.272 \\
$D_{\text {fog }}$ & -0.005 \\
$C$ & 1.225 \\
\hline
\end{tabular}

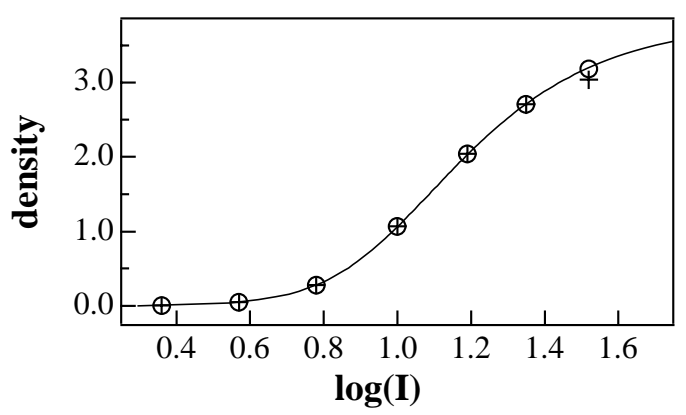

Fig. 3. The scanned density values of the northern sensitometer marks versus the corresponding $\log (I)$-values (plus sign). For the fit of the characteristic curve (line) we used the values indicated by circles. The highest measured density had to be slightly corrected for this purpose

and minor-axis $V$-band surface-brightness profiles of the bright early-type galaxies NGC 4696, 4709, and 4729 in Centaurus. These profiles were compared with azimuthally averaged profiles in the $B$-band we derived for these galaxies (cf. next subsection). As no significant radial colour gradients are observed for early-type galaxies in general (e.g. Reid et al. 1994), our profiles should fit between Bender's major and minor-axis profiles after applying an individual zero-point offset correction. This is illustrated in Fig. 4. Obviously a good transformation quality is achieved in the non-saturated regime from $\mu_{B}>$ $21.5 \mathrm{~B} \mathrm{arcsec}^{-2}(B-V \sim 1)$ down to $\mu_{B} \sim 25.0 \mathrm{~B} \operatorname{arcsec}^{-2}$. The accuracy at a fainter level, between $25.0 \mathrm{~B} \mathrm{arcsec}-2$ and $\sim 27.0 \mathrm{~B} \mathrm{arcsec}^{-2}$ (which relies mostly on the accuracy of the sky background estimation) can be judged from the linear shapes of the surface-brightness profiles of faint dwarfs shown in Fig. 5 .

Each individual zero-point offset is the sum of the calibration constant of our instrumental magnitude to the $B$-magnitude system and the $B-V$ galaxy colour which could be used to calibrate our magnitude system in principal. But neither $B_{\mathrm{T}}$ nor $B-V$ are very well known for the three galaxies (Sadler 1984; Poulain 1988; Dressler et al. 1991; Longo \& de Vaucouleurs 1983; de Vaucouleurs et al. 1991; Prugniel et al. 1993). 


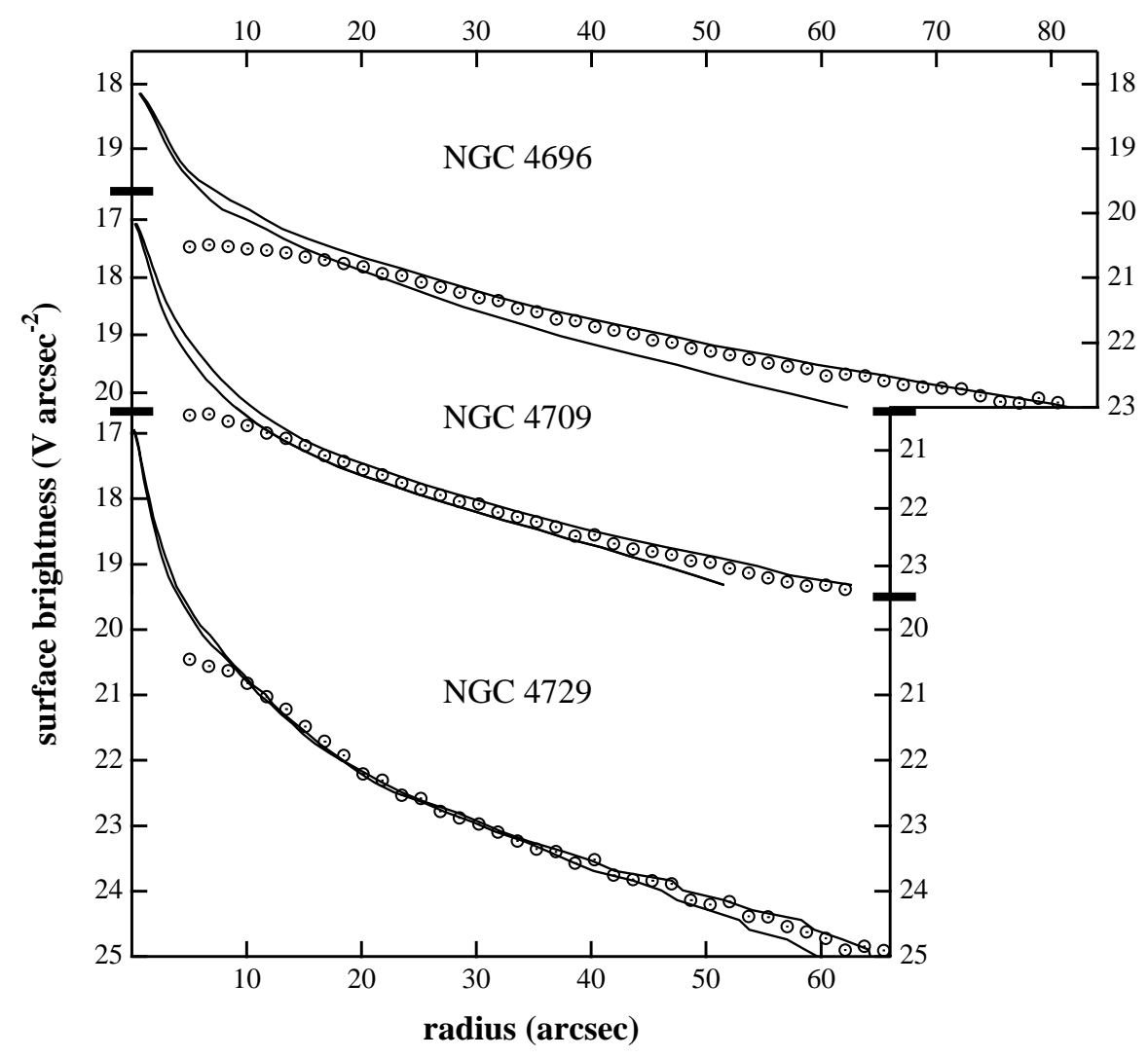

Fig. 4. The major and minor-axis surface-brightness profiles in the $V$-band for NGC 4969, 4709, and 4729 are shown as lines. Our azimuthally averaged surface-brightness profiles are added as dotted circles. The best-fitting offset from the $B$-band to the $V$-band magnitude system was determined individually for each galaxy

\subsection{Image reduction}

The neighbourhood of each cluster galaxy image was disentangled interactively from surrounding stars. To do this we used procedures developed in the image processing program MIDAS. If possible, stars projected on a galaxy surface were eliminated by taking advantage of the symmetric property of the galaxy. The pixels of such a star were substituted by the pixels lying on the point symmetrical opposite of the galactic centre. In cases where this technique was not applicable, e.g. the opposite pixels were also contaminated by stars or the galaxy showed asymmetrical appearance, the underlying galactic ground was modelled by fitting locally a second order 2D-polynomial.

After the galaxy image was star subtracted we determined for the galaxy a growth curve based on a visually selected centre. The pixel intensities were integrated in concentric round apertures of increasing radius in steps of 1 pixel $\left(=1.68^{\prime \prime}\right)$ outwards. Simultaneously, the sky intensity was subtracted so the growth curve became asymptotically flat for sufficiently large radii and a correct sky background determination. This first growth curve was used to define the maximum radius where all light of the galaxy is included and the noise of the background starts to domi- nate. A new galaxy centre was defined by the luminosityweighted first moment of all pixels within the maximum aperture and the second (final) growth curve $G C(r)_{\text {obs }}$ was established for the galaxy.

Apart from few cases this growth curve was smooth enough to derive the corresponding instrumental surfacebrightness profile:

$S B(r)=-2.5 \log \left(\left.\frac{\mathrm{d} G C(s)_{\mathrm{obs}}}{\mathrm{d} s}\right|_{s=r}\right)+$ const.

The profile was approximated analytically in three possible ways depending on the Hubble type of the galaxy. Classically, the light profiles of ellipticals or the spheroidal components of disk galaxies were described by the $r^{1 / 4}$-law (de Vaucouleurs 1948). A more general approach represents the generalized exponential law (Sérsic 1968): $I(r)=$ $I_{0} \exp (-r / \alpha)^{n}$ or $\mu(r)=\mu_{0}+1.086(r / \alpha)^{n}, \quad 0<n$, where the exponent remains free instead of being fixed at $n=1 / 4$. It has been shown recently (Caon et al. 1993; Graham et al. 1996) that this formula offers a much better approach to the observed profiles of elliptical galaxies. We were thus motivated to fit most of these profiles by this analytic form. The shape parameters $n$ are listed in the CCC. 
S0's and spirals consist of two different profile components. The inner bulge follows the de Vaucouleurs law as the ellipticals. The outer disk part shows a linear decay of the surface brightness corresponding to an exponent $n=1$ in the generalized exponential law. Consequently we fitted a two-component model at the profiles of these galaxies.

Profiles of some bright nucleated dwarf galaxies (dE, N and dS0, N) exhibit a clear bipartition. In these cases we approximated the profile by a two-component exponential model (Binggeli \& Cameron 1991). Generally, a classical exponential law was used for the dE's, dS0's, Im's, and BCD's as well as for some dE, N's and dS0, N's. Note that the nuclei of $\mathrm{dE}$, N's or dS0, N's in Centaurus have a typical size of $\leq 3^{\prime \prime}$. Therefore, the seeing effect and the discreteness of the scanning process can easily destroy the central light excess in the empirical surface-brightness profile.

The innermost radius limit for all fits was 3 arcsecs and a lower limit in instrumental surface-brightness was applied equivalent to $27 \mathrm{~B} \mathrm{arcsec}{ }^{-2}$. For some bright galaxies the centre of the image was saturated due to photographical limitation. Here, the fit was restricted to the undisturbed outer part of the profile starting at an equivalent surface brightness of $21.5 \mathrm{~B} \mathrm{arcsec}{ }^{-2}$.

The model-dependent parameters $\mu_{0}^{\exp }$ and $\alpha$ were derived from the pure exponential outer part of a profile. As the surface brightness scale is logarithmic, an exponential profile appears as a straight line. The linear extrapolation of the best-fitting line into the centre yields the central exponential surface brightness $\mu_{0}^{\exp }$. The exponential scale length $\alpha$ of the galaxy corresponds to the slope of this line.

Moreover, we determined three model-independent parameters for each galaxy: the instrumental total magnitude $m_{\text {inst }}$, the effective radius $r_{\text {eff }}$ (radius containing half of the total light), and the mean effective surface brightness $\langle\mu\rangle_{\mathrm{eff}}$. For those galaxies with no saturation problems in the surface-brightness profile we analysed the observed growth curve for this purpose. In particular, the asymptotic limit of the growth curve at maximum aperture defined the total instrumental intensity of the galaxy. For all other cases, the parameters were derived from the growth curve corresponding to the best-fitting surface brightness model.

As already mentioned above, there were few galaxies, mainly faint early-type dwarf galaxies, where the growth curve was so badly affected by sky background noise that a derivation of the surface-brightness profile would require to run a smoothing algorithm first. All structure parameters have been derived from the best-fitting model of an integrated pure exponential law into the noisy growth curve starting at $r>3^{\prime \prime}$ :

$G C(r)=2 \pi I_{0} \alpha^{2} \gamma[2,(r / \alpha)]$,

with $\gamma(a, x)=\int_{0}^{x} \exp (-s) s^{a-1} \mathrm{~d} s, I$ the intensity, and $\alpha$ the exponential scale length.
For illustration we present in Fig. 5 an arbitrarily chosen collection of eight calibrated surface-brightness profiles. In the upper left diagram the saturation effect due to the limits of the photographic plate is clearly visible. A fit of a generalized exponential law to the undisturbed outer part of the profile was used to extrapolate into the central region.

\subsection{Vignetting correction}

All our magnitudes were corrected for the geometrical vignetting effect of the UKSTU Schmidt telescope. We used the experimentally determined vignetting function (cf. Dawe \& Metcalfe 1982; Tritton 1983). In general, the correction factor varies on the photographic plate from 1.0 at the centre to 1.25 at 4 degrees off axis. At the position of the Centaurus cluster on plate 323J a typical correction of $3 \%$ had to be applied.

Because of the difficulties to quantify the effect of the desensitization of hypersensitized plates ("Malin effect"), no correction was applied for this additional effect. It has been studied in detail by Campbell (1982) and a maximum error of less than 0.04 is quoted by Dawe \& Metcalfe (1982).

\subsection{Magnitude zero point determination}

The goal was to transform the instrumental magnitudes $m_{\text {inst }}=-2.5 \log \left(I_{\text {tot }}\right)$ into CCD $B$-magnitudes. For this purpose two sets of independent data were used from the literature. A selected sample of bright galaxies was taken from the Surface Photometry Catalogue of the ESOUppsala Galaxies (Lauberts \& Valentijn 1989 hereafter LV). These magnitudes proved to be in good agreement with the standard RC3 system (Paturel et al. 1994). The selection criteria we applied to find those galaxies which are most reliable for the calibration were twofold: First, the central part of the surface-brightness profile must not be affected by saturation effects. Second, our derived value for $2 r_{\text {eff }}$ should not differ too much from the effective diameter $A_{\mathrm{e}}^{B}$ given by LV. The motivation for the second constraint is based on an existing discrepancy between LV and other authors about the $A_{\mathrm{e}}^{B}$ values of an early-type galaxy sample (cf. Fig. 9 in LV). Based on this uncertainty we arbitrarily fixed the allowed maximum deviation between $A_{\mathrm{e}}^{B}$ and $2 r_{\text {eff }}$ at $15 \%$. By this selection we expect to take into consideration only the uncontroversial galaxy data of both studies. The two constraints were met by 10 galaxies.

$\mathrm{LV}$ offers only few galaxies fainter than $B_{\mathrm{T}} \sim 16$. Thus, we supplemented the calibrator sample with a CCD-based data set of 22 dwarf galaxies (Bothun et al. 1989 hereafter BCS89). This second sample covers well the magnitude interval $15.5<B_{\mathrm{T}}<19.0$ and will improve the calibration accuracy at the faint end. The two data sets of LV and BCS89 galaxies are collected in Table 4. 

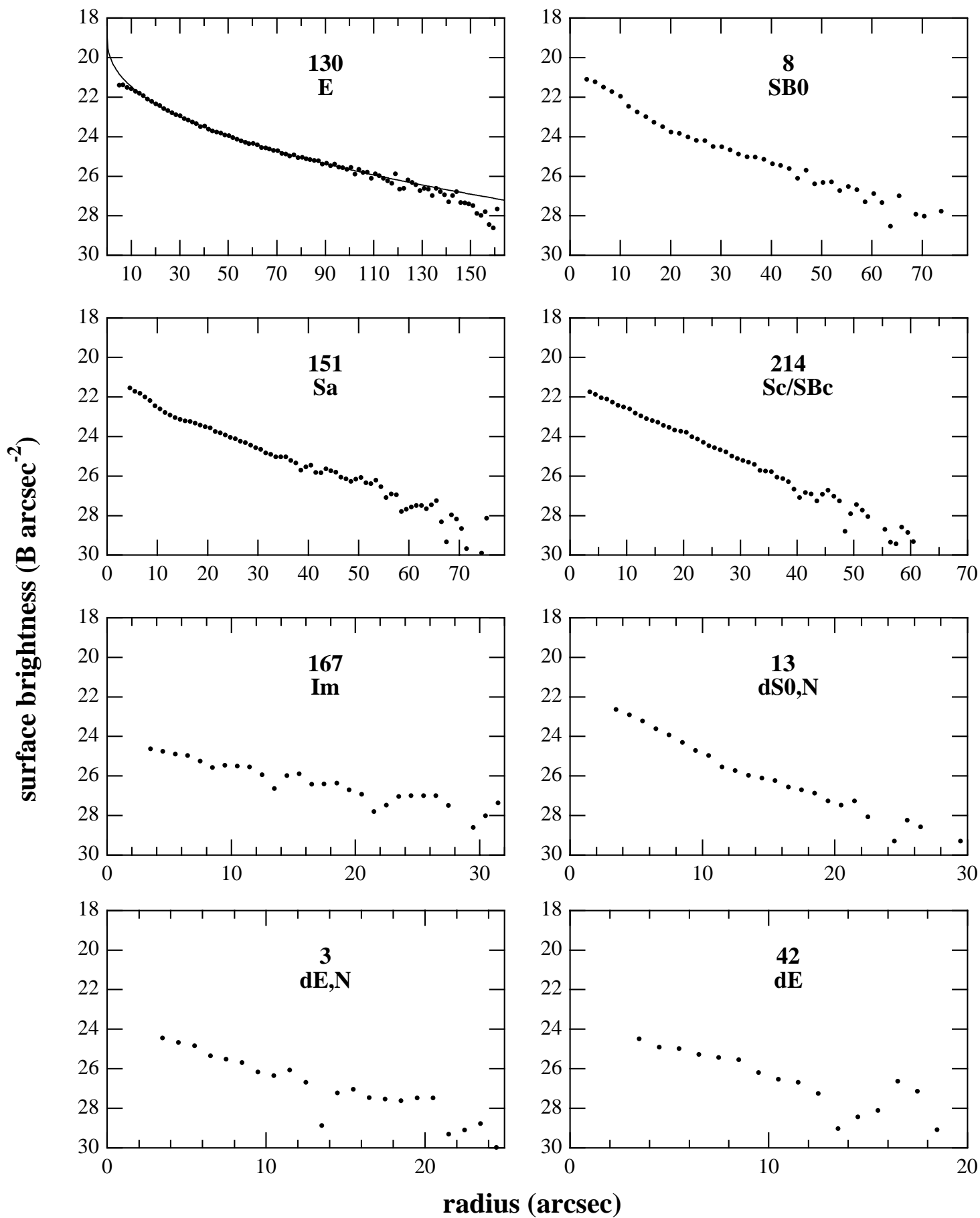

Fig. 5. Selected non-smoothed mean surface-brightness profiles in order of decreasing total apparent magnitudes are shown. The CCC numbers are listed, along with the galaxy classifications. The points are plotted at $1^{\prime \prime}$ intervals. Notice the saturation effect in the inner part of the CCC130 profile and its correction by the best-fitting model 
Table 4. Calibration data

\begin{tabular}{rccc||rcccc|rccc}
\hline ESO & $m_{\text {inst }}$ & $B_{\mathrm{T}}$ & $(B-R)_{\mathrm{T}}$ & $\mathrm{G}$ & $m_{\text {inst }}$ & $V$ & $(B-V)$ & $\mathrm{G}$ & $m_{\text {inst }}$ & $V$ & $(B-V)$ \\
\hline $322-087$ & -8.81 & 14.68 & 1.37 & 36 & -5.68 & 16.96 & 1.08 & 75 & -5.18 & 16.85 & 0.98 \\
$322-090$ & -8.94 & 14.56 & 1.34 & 41 & -5.82 & 16.69 & 1.02 & 76 & -5.45 & 17.22 & 0.96 \\
$322-093$ & -7.45 & 16.11 & 0.95 & 46 & -5.80 & 17.14 & 1.04 & 77 & -5.33 & 17.14 & 0.94 \\
$322-096$ & -8.49 & 15.02 & 1.41 & 48 & -5.05 & 17.49 & 1.05 & 78 & -5.29 & 17.44 & 0.86 \\
$323-006$ & -7.79 & 15.74 & 1.11 & 54 & -4.98 & 17.51 & 0.99 & 81 & -4.82 & 17.97 & 1.00 \\
$323-018$ & -8.83 & 14.84 & 1.45 & 55 & -6.16 & 16.50 & 1.01 & 85 & -5.98 & 16.86 & 0.99 \\
$323-020$ & -8.77 & 15.01 & 1.22 & 62 & -6.90 & 15.49 & 0.96 & 94 & -7.64 & 14.85 & 1.02 \\
$323-022$ & -9.99 & 13.47 & 1.55 & 66 & -5.31 & 17.05 & 0.96 & 95 & -5.26 & 17.33 & 1.00 \\
$323-038$ & -9.13 & 14.37 & 1.54 & 67 & -6.59 & 16.24 & 0.85 & 97 & -5.38 & 17.01 & 1.07 \\
$323-042$ & -9.42 & 14.10 & 1.30 & 69 & -6.44 & 16.20 & 1.00 & 101 & -5.46 & 17.24 & 0.91 \\
& & & & 74 & -5.80 & 16.92 & 0.97 & 103 & -4.35 & 18.17 & 0.90 \\
\hline
\end{tabular}

ESO: Laubert \& Valentijn (1989); G: Bothun et al. (1989).

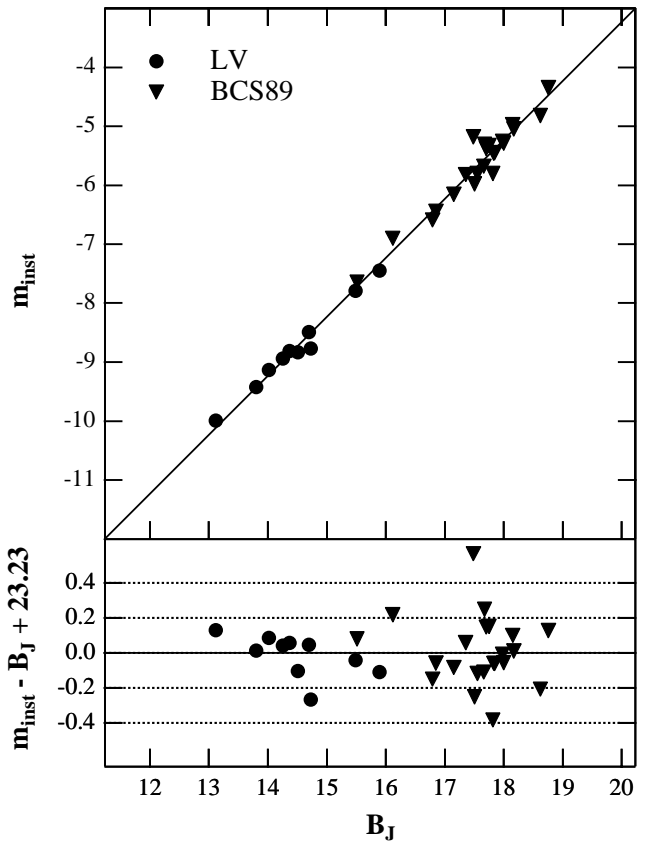

Fig. 6. The upper graph shows the calibration diagram based on 32 selected galaxies in common with LV (circle) and BCS89 (triangle). The indicated line is not a fit, but a line with unity slope representing a perfect relation between two magnitude systems. Obviously, this line is very well approximated by the data points. The residual diagram for our $B_{\mathrm{J}}$-magnitudes is given in the bottom panel

We converted the CCD $B$-magnitudes from the BCS89 sample to the bandpass IIIa-J $\left(B_{\mathrm{J}}\right)$ using the colour equation $B_{\mathrm{J}}=B-0.35(B-V)$ as determined by Metcalfe et al. (1995). The approximately equivalent relation $B_{J}=$ $B_{\mathrm{IIa}-\mathrm{O}}-0.23(B-R)_{\mathrm{IIa}-\mathrm{O}}$ was applied on the $B_{\mathrm{IIa}-\mathrm{O}^{-}}$ magnitudes of LV. The calibration diagram is shown in Fig. 6. A linear fit quantifies the transformation rule to be

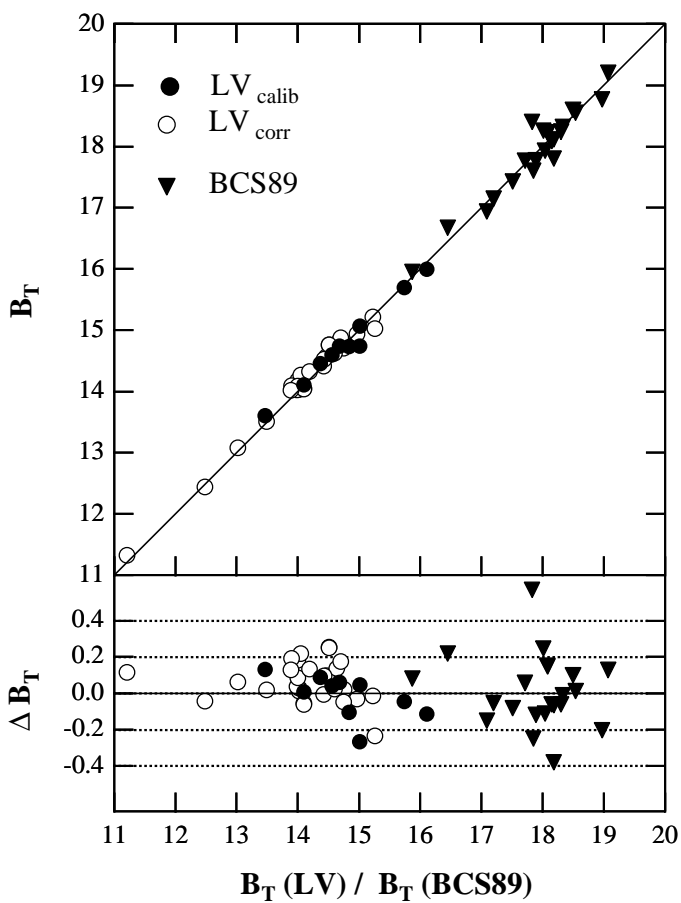

Fig. 7. $B_{\mathrm{T}}$-magnitudes of all galaxies in common with LV. Filled circles are galaxies used for the magnitude calibration. Open circles are galaxies required individual corrections for central saturation effects

$m_{\text {inst }}=-23.14( \pm 0.33)+0.99( \pm 0.02) \cdot B_{\mathrm{J}}$. If restricted to the magnitude range of particular interest between $B_{\mathrm{J}} \sim 11$ and 21 , the fitted line differs at most 0.04 from a transformation rule with a unity slope. Based on this good agreement we assumed an underlying relation $m_{\text {inst }}=B_{\mathrm{J}}+Z P$ and determined the zero point with $Z P=-23.23$. This value was applied to transform our instrumental magnitudes into $B_{\mathrm{J}}$-magnitudes. The rms scatter in Fig. 6 is $0 .{ }^{\mathrm{m}} 18$ which translates to an 
uncertainty of the calibration zero point of 0.03 . Subsequently all $B_{\mathrm{J}}$-magnitudes were converted into $B$ magnitudes using the colour equations given above where the measured colours were available. The average colour of the BCS sample $\langle(B-V)\rangle=0.98$ was used where colours had not been measured. The last value is about equivalent to $B-R=1.49$ and comparable to the mean $B-R$ colour of $1.46( \pm 0.21)$ for all LV galaxies in common (not only the calibrators).

A weakness of photographic plates is the relatively small dynamic range they cover. Long-time exposures are leading to saturation effects on central parts of bright galaxies (cf. Fig. 4). The surface-brightness profile of such a galaxy had to be extrapolated to the centre in a way as described before. To get an idea how well this procedure worked, we compare in Fig. 7 the $B_{\mathrm{T}}$-magnitudes of the 10 uncorrected LV calibrators with those of other 28 galaxies in common with $\mathrm{LV}$ which required a saturation correction. We found good statistical agreement between the two samples, $\left\langle\Delta B_{\mathrm{T}}\right\rangle_{\text {uncorr }}=0.02( \pm 0.12)$ and $\left\langle\Delta B_{\mathrm{T}}\right\rangle_{\text {corr }}=0.06( \pm 0.11)$ with only a slight systematic error for the corrected sample of $0.06( \pm 0.02)$. This result shows the reliability of our correction procedure within the expected magnitude error as described below. Furthermore, we note that the complete LV sample covers a wider colour range $0.8<(B-R)<1.7$ than the calibrators. Apparently the transformation rule between the instrumental and $B$-magnitude systems seems to work well for all these galaxies.

\subsection{Accuracy of galaxy parameters}

The photometric accuracy of the instrumental magnitudes was estimated from the residuals illustrated in the bottom diagram of Fig. 7. First of all it is satisfactory to see that there are no systematic magnitude differences between our magnitudes and those of the bright and faint calibrator samples, respectively: $\left\langle\Delta B_{\mathrm{T}}\right\rangle_{\mathrm{LV}}=0.02( \pm 0.12)$ and $\left\langle\Delta B_{\mathrm{T}}\right\rangle_{\mathrm{BCS} 89}=0.00( \pm 0.19)$. We conclude that the global errors of our $B_{\mathrm{T}}$-magnitudes for brighter galaxies are comparable to the typical uncertainty $\sim 0.2$ for $\mathrm{LV}$ magnitudes (Paturel et al. 1994). We further propose an error of \pm 0.3 for galaxies fainter than $B_{\mathrm{T}} \sim 17$.

BCS89 published the model parameters $\alpha$ and $\mu_{0}^{\exp }$ for their 22 faint galaxies which can be used to estimate the accuracy of other structure parameters. In Fig. 8 we plot the scale length and central exponential surface brightness data of our studies. Obviously, good agreement is found for $\log (\alpha)$. The typical error is $\sigma_{\log (\alpha)}=0.12$ and no systematic offset is evident in the data. In the case of $\mu_{0}^{\text {exp }}$ we find a mean difference of $\Delta \mu_{0}^{\exp }=0.12$ with a standard deviation of $\sigma_{\mu_{0}^{\exp }}=0.26$.

Most of the dwarf elliptical galaxies exhibit in first order a pure exponential surface-brightness profile (e.g. Binggeli \& Cameron 1991) which relate the effective radius and the scale length by the equality $r_{\mathrm{eff}}=1.667 \alpha$.
Taking advantage of this formula we estimate the error for $\log \left(r_{\text {eff }}\right)$ to be roughly the same as that of $\log (\alpha)$. The error of $\langle\mu\rangle_{\text {eff }}$ may be \pm 0.4 combining the uncertainties of $B_{\mathrm{T}}$ and $\log \left(r_{\mathrm{eff}}\right)$.

Acknowledgements. This paper is part of the $\mathrm{PhD}$ thesis of one of the authors (HJ). He would like to thank his supervisors G.A. Tammann and Bruno Binggeli for their interesting ideas and invaluable advice. We are most grateful to Ralf Bender who made available to us galaxy surface-brightness profiles as well as to the ESO head quarter in Garching for giving access to the PDS plate-scanning machine. We thank Ken Freeman and the referee Dr. E. Bertin for having read the manuscript and having improved the origin version by many suggestions. HJ thanks the Swiss National Science Foundations for financial support.

\section{Appendix}

\section{A. The Centaurus Cluster Catalogue}

The Centaurus Cluster Catalogue CCC is available in electronic form at the CDS via anonymous ftp 130.79.128.5. It contains all 296 galaxies detected in the survey area and classified with a membership probability of $50 \%$ or more. For each galaxy we give a set of derived fundamental parameters. The data are organized as follows:

Column (1). Centaurus Cluster Catalogue number.

Columns (2) $+(3)$. Right ascension and declination (1950.0). To determine the equatorial coordinates of the galaxies we drew on the star positions of the Hubble Space Telescope Guide Star Catalogue (Russel et al. 1990). For each of the 16 digitized images a well distributed sample was selected of at least 15 guide stars. Their coordinates define a reference system for the specific image and were used to convert the $X-Y$ coordinates of the galaxies into equatorial coordinates. As an internal accuracy check we further selected on each image a second sample of 10 other guide stars. The rectangular coordinates of these stars were determined and transformed to equatorial coordinates in a similar way as those of the galaxies. The deviations of our achieved coordinates from their GSC coordinates gave the mean position error. For all 16 images of the Centaurus region this value is less than 1 arcsec.

Column (4). Morphological type. The galaxy classification follows primary the precepts of Sandage (1961) and Sandage \& Binggeli (1984) in the case of the giant galaxies and the dwarfs, respectively. The main giant classes are: E, S0, Sa, Sb, Sc, Sd, and $\mathrm{Sm}$ with intermediate classes E/S0, S0/a, Sab, etc. Barred lenticulars or spirals are indicated as SB. Following Hubble, E and S0 galaxies are classified according to their flattening. Thereby, the minor-to-major axis ratio $b / a$ of a galaxy is coded as Ex or S0 $(x)$ where $x:=10(1-b / a)$. Dust features in a S0 are noted by a subindex 1, 2 or 3 according to their strength 

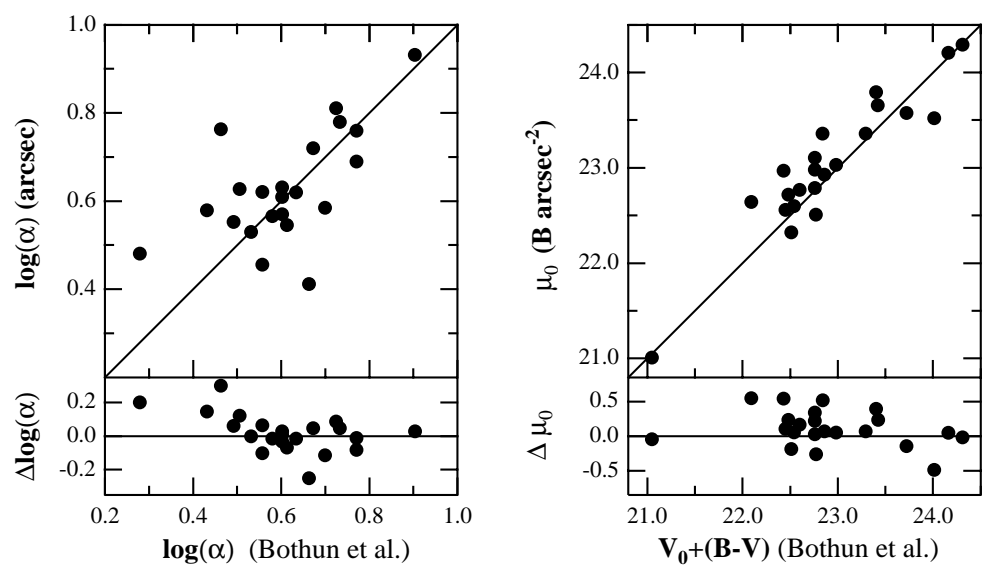

Fig. 8. Comparison of the model- depending parameters $\log (\alpha)$ and $\mu_{0}^{\text {exp }}$ for a sample of early-type dwarfs in common with BCS89. The solid lines represent perfect agreement between the two data sets

(Sandage 1961). The dwarf classes are: Im, BCD, dE, and dS0. Depending on the presence or absence of a central nucleus, a dE or dS0 has been added by a "N". If the uncertainty in the classification of a particular galaxy is larger than within a subtype it was marked by a symbol. Errors within the same Hubble type are indicated by ":" or by "?" in the case of an even larger uncertainty.

Column (5). Cluster membership probability. $1=$ definite member $(100 \%), 2=$ likely member $(75 \%), 3=$ possible member $(50 \%)$.

Column (6). Total apparent blue magnitude $B_{\mathrm{T}}$. The magnitudes are not corrected for galactic absorption into the direction of the Centaurus cluster which is still not very well known. Published values are in the range between $A_{B}=0.23$ (Sandage 1973) and 0.92 (Bothun et al. 1989). A good compromise seems to be 0.42 based on HI column density observations (Burstein \& Heiles 1982, 1984).

Column (7). Quality of the derived growth curve and surface-brightness profile. $1=$ the profile is not disturbed by a central saturation effect, nor was a smoothing process necessary due to a noisy background, $2=$ a weak saturation correction was applied (effect $<0.1$ in $B_{\mathrm{T}}$ ) or the curve was weakly smoothed. $3=$ a substantial saturation correction (effect $>0 \mathrm{~m}^{\mathrm{m}} 1$ in $B_{\mathrm{T}}$ ) or smoothing was needed. $4=$ growth curve was reliable only to determine the total apparent magnitude $B_{\mathrm{T}}$.

Column (8). Mean effective surface brightness $\langle\mu\rangle_{\text {eff }}$ in $\mathrm{B} \operatorname{arcsec}^{-2}$.

Column (9). Logarithm of the mean effective radius $r_{\text {eff }}$ (radius containing half of total light) in arcsec.

Column (10). Central exponential surface brightness $\mu_{0}$ in $\mathrm{B} \mathrm{arcsec}^{-2}$.

Column (11). Logarithm of exponential scale length $\alpha$ in $\operatorname{arcsec}$.

Column (12). Exponent of the generalized exponential profile (shape parameter).
Column (13). Heliocentric radial velocity in $\mathrm{km} \mathrm{s}^{-1}$. The indicated integer represents three different references: 1 = Dickens et al. (1986), 2 = Lucey \& Carter (1988), $3=$ Stein (1994).

Column (14). Various names by which each galaxy is known. The letters indicate the following 4 catalogues: B (Bothun et al. 1989), D (Dressler 1980), DCL (Dickens et al. 1986), 6 digit number (Lauberts 1982). The references for NGC galaxies are given in Table 5 .

Table 5. Finding list of NGC galaxies

\begin{tabular}{rl|rl|rl}
\hline CCC & NGC & CCC & NGC & CCC & NGC \\
\hline 8 & 4683 & 65 & 4696 & 211 & 4730 \\
25 & $4696 \mathrm{C}$ & 122 & 4706 & 226 & 4743 \\
43 & $4696 \mathrm{D}$ & 130 & 4709 & 227 & 4744 \\
50 & $4696 \mathrm{E}$ & 204 & 4729 & & \\
\hline
\end{tabular}

Comment: The two galaxies CCC 88 and $\mathrm{CCC} 212$ were strongly disturbed by bright stars in their very near neighbourhood. No satisfactory reduction of these images was possible and the $B_{\mathrm{T}}$-magnitudes were estimated by eye. Other structure parameters have not been derived.

\section{References}

Bender R., 1994 (private communication)

Binggeli B., Cameron L.M., 1991, A\&A 252, 27

Binggeli B., Cameron L.M., 1993, A\&AS 98, 297

Binggeli B., Popescu C.C., Tammann G.A., 1993, A\&AS 98, 275

Binggeli B., Sandage A., Tammann G.A., 1985, AJ 90, 1681

Binggeli B., Sandage A., Tarenghi M., 1984, AJ 89, 64 
Binggeli B., Tammann G.A., Sandage A., 1987, AJ 94, 251

Binggeli B., Popescu C.C., Tammann G.A., 1993, A\&AS 98, 275

Bothun G.D., Caldwell N., Schombert J.M., 1989, AJ 98, 1542 $(=\mathrm{BCS} 89)$

Bowen I.S., and Vaughan Jr. A.H., 1973, Appl. Opt. 12, 1430

Burstein D., Heiles C., 1982, AJ 87, 1165

Burstein D., Heiles C., 1984, ApJS 54, 33

Campbell A.W., 1982, Observatory 102, 195

Caon N., Capaccioli M., D‘Onofrio M., 1993, MNRAS 265, 1013

Dawe J.A., Metcalfe N., 1982, Proc. Astr. Soc. .Austr. 4, 466

de Vaucouleurs G., 1948, Ann. d'Astrophys. 11, 247

de Vaucouleurs G., de Vaucouleurs A., Corwin H.G., Buta R., Paturel G., Fouqué P., 1991, Third Reference Catalogue of Bright Galaxies. Springer, New York $(=\mathrm{RC} 3)$

Dickens R.J., Currie M.J., Lucey J.R., 1986, MNRAS 220, 679

Dressler A., 1980a, ApJ 236, 351

Dressler A., 1980b, ApJS 42, 565

Dressler A., Faber S.M., Burstein D., 1991, ApJ 368, 54

Drinkwater M.J., Currie M.J., Young C.K., Hardy E., Yearsley J.M., 1996, MNRAS 279, 595

Ferguson H.C., 1989, AJ 98, 367

Ferguson H.C., Sandage A., 1988, AJ 96, 1520

Ferguson H.C., Sandage A., 1991, AJ 101, 765

Freeman K.C., 1970, ApJ 160, 811

Graham A., Lauer T.R., Colless M., Postman M., 1996, ApJ 465,534

Jerjen H., Binggeli B., 1997, (in preparation) (Paper IV)

Lauberts A., 1982, The ESO/Uppsalla Survey of the ESO(B) Atlas, European Southern Observatory

Lauberts A., Valentijn E.A., 1989, The Surface Photometry Catalogue of the ESO-Uppsala Galaxies, ESO, Garching bei München (=LV89)

Llebaria A., Figon P., 1981, in: Proceedings on Astronomical
Photography, Heudier J.-L. et al. (eds.) Centre National Recherche Scientifique, Nice, p. 25

Longo G., de Vaucouleurs A., 1983, A General Catalogue of Photoelectric Magnitudes and Colors in the $U, B, V$ System, Monographs in Astronomy No.3, University of Texas

Lucey J.R., Dickens R.J., Dawe J.A., 1980, Nat 285, 305

Lucey J.R., Currie M.J., Dickens R.J., 1986, MNRAS 222, 427

Lucey J.R., Carter D., 1988, MNRAS 235, 1177

Lynden-Bell D., Faber S.M., Burstein D., Davies R.L., Dressler A., Wegner G., 1988, ApJ 326, 19

Metcalfe N., Fong R., Shanks T., 1995, MNRAS 274, 769

Paturel G., Bottinelli L., Gouguenheim L., 1994, A\&A 286, 768

Poulain P., 1988, A\&AS 72, 215

Prugniel P., Bica E., Klotz A., Alloin D., 1993, A\&AS 98, 229

Reid N., Boisson C., Sansom A.E., 1994, MNRAS 269, 713

Russel J.L., Lasker B.M., McLean B.J., Sturch C.R., Jenkner H., 1990, AJ 99, 2059

Sadler E.M., 1984, AJ 89, 34

Sandage A., 1961, Hubble Atlas of Galaxies, Carnegie Institution of Washington Publication No. 618, Carnegie Institution of Washington, Washington DC

Sandage A., 1973, ApJ 183, 711

Sandage A, Tammann G.A., 1981, A Revised Shapley-Ames Catalogue of Bright Galaxies, Carnegie Institution of Washington Publication No. 635. Carnegie Institution of Washington, Washington DC

Sandage A., Binggeli B., 1984, AJ 89, 919

Sandage A., Binggeli B., Tammann G.A., 1985a, AJ 90, 395

Sandage A., Binggeli B., Tammann G.A., 1985b, AJ 90, 1759

Sérsic J.-L., 1968, Atlas de galaxias australes, Observatorio Astronomica, Cordoba

Stein P., 1994, PhD thesis, University of Basel

Tritton S., 1983, UKSTU Handbook, Science and Engineering Research Council, Royal Observatory, Edinburgh 